\title{
AUDIOVISUAL EUROPEO, DIVERSIDAD CULTURAL Y MERCADO GLOBAL: ANÁLISIS DE LAS ACTUACIONES EXTERIORES DE LA POLÍTICA AUDIOVISUAL DE LA UNIÓN EUROPEA ${ }^{1}$
}

\author{
Dra. Carmina Crusafón \\ (Universidad Autónoma de Barcelona) \\ Carmina.Crusafon@uab.es
}

\begin{abstract}
Resumen: La presencia global del audiovisual europeo se ha convertido en una prioridad para la Unión Europea. Bajo el primer mandato de la Comisión Barroso, las autoridades comunitarias han empezado a desarrollar un conjunto de actuaciones de carácter exterior dentro del ámbito de la política audiovisual europea. Se trata de poner al audiovisual entre las prioridades de la agenda exterior de la UE bajo un doble prisma: el de buscar nuevos mercados para las obras europeas y a su vez, promocionar la diversidad cultural. En este artículo se analizarán las actuaciones exteriores comunitarias y se evaluará su dinámica predominante (industrial y/o cultural).
\end{abstract}

Palabras clave: Audiovisual, Unión Europea, política audiovisual, diversidad cultural, cooperación internacional.

Abstract: Global presence of European audiovisual has become a priority for the European Union. Under Barroso's Commission first mandate, European authorities have undertaken some actions to emphasize the external dimension of the EU audiovisual policy. Its aim is for audiovisual to be right in the centre of EU global agenda under double perspective: looking for new markets for European works and in turn, promoting cultural diversity. This paper will analyze main EU external actions in the audiovisual field and it will evaluate their prevalent dynamics (either industrial and / or cultural).

Keywords: Audiovisual, European Union, Audiovisual policy, Cultural Diversity, International Cooperation.

\footnotetext{
${ }^{1}$ Este artículo es el resultado de una estancia de investigación realizada en el SMIT Research Centre de la Vrije Unviersiteit Brussel (Bélgica) y fruto de las conversaciones mantenidas con funcionarios de la Comisión Europea, académicos y representantes de otras instituciones europeas implicadas en la política audiovisual europea.
} 
244 Audiovisual europeo, diversidad cultural y mercado global: Análisis de las actuaciones exteriores de la política audiovisual de la unión europea

\section{INTRODUCCIÓN}

$\mathrm{E}$ I escenario global actual presenta una nueva configuración geopolítica mundial, marcada por una necesidad imperante de los diferentes actores, entidades nacionales y supranacionales, de posicionarse para asegurarse un lugar en las decisiones mundiales. El protagonismo de las entidades supranacionales y los foros multilaterales ha obligado a la Unión Europea a empezar a desplegar de forma más explícita y prioritaria su acción exterior.

De hecho, la dimensión exterior de la política audiovisual es una muestra más de un tema clave para la UE. Esto es, "el poder de influencia de Europa en el mundo, que se ha extendido de forma cada vez más visible desde el año 2004 con la ampliación a la Europa de los 25" (Foucher, 2009). Este crecimiento de la acción exterior de la UE se puede enmarcar en un nuevo estadio del proceso de espacialización, explicado desde la Economía Política. Como nos señala el investigador canadiense Vincent Mosco (2009: 256), podemos concebir la espacialización "como un medio para entender la relación de las geometrías de poder con los procesos del espacio, en particular, el espacio a través del cual la comunicación fluye". En esta nueva etapa, las geometrías de poder están variando y tomar el caso del sector audiovisual es un buen ejemplo de lo que realmente significan estos cambios.

Como complemento de la nueva configuración del escenario global, tres razones concretas avalan el protagonismo de la acción exterior de la Unión Europea. Primera, la evaluación de las acciones de la Comisión Barroso I (Crusafon, 2009a) pone de relieve que en la Política Audiovisual Europea (PAE), a partir del año 2008, existe una voluntad de ampliar la acción exterior hacia el ámbito global y en cierto modo, exportar el modelo europeo de política audiovisual. Segunda, la aprobación final del Tratado de Lisboa otorga un mayor protagonismo a la acción exterior de la UE. En concreto, el capítulo 1 del Tratado incluye unas disposiciones generales relativas a la política exterior de la Unión. Tercera, el escenario geopolítico global pone en marcha un nuevo orden mundial, con un nuevo multilateralismo creciente, donde la UE debe encontrar su papel y rol, combinándolo con las acciones de sus estados miembros, así como definiendo sus relaciones con Estados Unidos y los países emergentes. Todo ello para determinar si realmente lo que prima en las Relaciones Internacionales es el bilateralismo o se impone un renovado multilateralismo (Crusafon, 2009b).

Este análisis de la acción exterior de la UE supone también la evaluación de la existencia de un modelo europeo aplicado al caso de la política audiovisual. Este objetivo va a suponer tratar un tema de cierta complejidad que parte de la misma definición de lo que se entiende por europeo, ya que existen algunas dudas por resolver. Como señala el profesor francés Foucher (2009), “¿cómo se puede actuar en el mundo si se ignora los limites del lugar del que se habla?" y además, habrá que determinar si las bases de lo europeo se fijan en la identidad supranacional y en el protagonismo de la dimensión cultural frente a la económica. 
En este sentido, en los últimos años la Unión Europea ha desarrollado una política común en el sector audiovisual que se está convirtiendo en un modelo de referencia en el entorno internacional. Dos elementos han contribuido a consolidar la política europea audiovisual en el ámbito global: el primero, la ampliación interna del propio modelo como resultado de la adhesión a la UE de nuevos estados miembros; y el segundo, el desarrollo de una dimensión exterior en foros multilaterales y bilaterales que permiten dar visibilidad y protagonismo a la propuesta europea.

Este artículo tiene como objetivo analizar las actuaciones exteriores de la política audiovisual europea en los últimos dos años (2007-2009), porque ha incrementado su protagonismo en la acción comunitaria, especialmente de la Comisión Europea (De Cockborne y Trettenbrein, 2008). En este análisis, se evaluará el papel que juega la defensa de la diversidad cultural a través de la Convención de la UNESCO (2005), y qué elementos de mercado prevalecen en esta acción comunitaria. Todo ello para poder determinar la primacía de la dualidad culturaleconómica en la dimensión exterior o bien confirmar un predominio de la parte de mercado sobre la cultura.

Para poder explicar este protagonismo de la acción exterior de ámbito global, se realizará, en primer término, un repaso de las bases que permiten que tenga mayor presencia la dimensión exterior de la PAE. A continuación, se explicará el marco de actuación, áreas y principales conceptos utilizados. En tercer lugar, se analizan los tres principales instrumentos: los protocolos de cooperación cultural; el programa MEDIA INTERNATIONAL y MUNDUS; y la cooperación de carácter más técnico. Para concluir con una propuesta de definición sobre la acción exterior de la PAE y sobre la exportación del modelo europeo a nivel global.

\section{LAS BASES PARA IMPLEMENTAR LA DIMENSIÓN EXTERIOR DE LA PO- LIITICA AUDIOVISUAL EUROPEA}

En primer término, es necesario preguntarse la siguiente cuestión: ¿Es algo novedoso la dimensión exterior de la PAE? La respuesta sería negativa en tanto que se trata de un planteamiento común a todas las políticas comunitarias. Lo novedoso está en la dimensión global que ha tomado durante el primer mandato de la Comisión Barroso (2004-2009), abarcando el conjunto del planeta para ayudar a la UE a consolidarse como uno de los actores clave en el escenario global, donde diferentes instituciones van tomando protagonismo en la gobernanza mundial (Jakubowicz, 2007: 207-220). Cambian los parámetros de la geopolítica mundial, donde hay una dicotomía entre el nuevo multilateralismo y el bilateralismo, haciendo de la política supranacional un elemento central en las dos últimas décadas (Flew, 2007: 213). Hay un discurso donde predomina el primero, pero en el análisis de las acciones se observa un bilateralismo creciente, influencia de la política estadounidense, donde la UE intenta conseguir un posicionamiento relevante acompañado de un buen número de aliados. 
Audiovisual europeo, diversidad cultural y mercado global: Análisis de las actuaciones exteriores de la política audiovisual de la unión europea

Si repasamos la historia de la PAE desde sus inicios (en los años 80), nos damos cuenta de que han existido algunos episodios donde la dimensión exterior ha tenido mayor presencia, por ejemplo, a principios de los 90 en las negociaciones del GATS (1990-1993) y en los acuerdos multilaterales de inversión en el seno de la OCDE. En ambos el audiovisual fue uno de los temas clave de la negociación (Crusafon, 1999: 548-556; Chakravarty y Sarikakis, 2006: 103-106); después los esfuerzos se fueron centrando en la ampliación de la Comunidad Europea, con la incorporación de nuevos estados miembros y un interés hacia la política de vecindad, especialmente con los países del Mediterráneo.

Los precedentes más recientes a esta actuación comunitaria hay que buscarlos en dos momentos relevantes para las instituciones europeas. Por un lado, la Agenda de Lisboa, fruto del Consejo Europeo de 23-24 marzo de 2000, donde se señala al audiovisual como "una poderosa herramienta para las relaciones internacionales y como un componente esencial para consolidar y mejorar la cooperación internacional cultural y promover la diversidad cultural" (Comisión Europea, 2009c: 3). Por otro, esta agenda se complementará con la aprobación y ratificación de la Convención sobre la Diversidad Cultural de la UNESCO, adoptada en octubre 2005 y que no entraría en vigor en la UE hasta marzo de 2007. Posteriormente, la Comisión Europea presentó una comunicación, en junio de 2006, bajo el título "Europa en el mundo", en la que se establecía la relación entra las políticas internas y externas y la necesidad de aprovechar las oportunidad de los mercados emergentes. A ello se unirán las directrices que marca el documento conocido como "Agenda Europea para la Cultura en un mundo globalizado" (Consejo de la UE, 2007). Este documento señala tres objetivos comunes: la diversidad cultural y el diálogo intercultural; la cultura como catalizador para la creatividad; y la cultura como componente claro de las Relaciones Internacionales. Sobre el último, se establece la cultura como elemento vital de la acción exterior, bajo el paraguas de la Convención UNESCO, promocionando un rol cultural más activo de la UE en las Relaciones Internaciones. Señala también la necesidad de integrar la dimensión cultural como elemento vital para los acuerdos de la UE con países y regiones socios (Humphreys, 2008a: 167-69). Como afirma la investigadora Divina Frau-Meigs (2008: 107), "la UE se ha comprometido con la UNESCO para jugar un papel de liderazgo en la promoción de la cultura al incluirla en todos sus proyectos de desarrollo".

En el caso más específico de la política audiovisual europea, la dimensión exterior ha tomado un protagonismo especial a partir del año 2008. En concreto, la "Declaración de Cannes", presentada durante el día europeo en el Festival de Cine de Cannes (19 de mayo 2008), pone de manifiesto la necesidad de dar mayor dimensión externa a las actuaciones comunitarias para no desaprovechar las oportunidades que brinda la creciente internacionalización de los mercados audiovisuales (Comisión Europea, 2008c). A partir de la "Declaración de Cannes" se han puesto en marcha algunas iniciativas que corroboran la tendencia a dar mayor protagonismo a la dimensión exterior global. Por un lado, la iniciativa del Parlamento europeo de ampliar el ámbito de acción del programa MEDIA para 
reforzar la cooperación audiovisual entre los profesionales de la industria europea y los profesionales de terceros países. Por otro, relacionar el desarrollo de la cooperación audiovisual con aquellos países que compartan una visión común sobre la diversidad cultural y en particular, a las posibilidades ofrecidas por el artículo 1 de la Directiva de Servicios de Comunicación Audiovisual; así como recomendar tener en cuenta en las actuaciones los desequilibrios entre los intercambios al igual que a los beneficios mutuos (Burri- Nenova, 2007). Ese mismo año, en noviembre, las conclusiones del Consejo Europeo, bajo presidencia francesa, se centraron en la promoción de la diversidad cultural y del diálogo intercultural en las relaciones exteriores de la UE y de sus Estados Miembros. En ellas, se indica que "el lugar de Europa en el mundo, desde un punto de vista artístico, intelectual y científico, depende de una considerable extensión en el dinamismo de sus trabajos creativo cultural y en sus intercambios culturales con terceros países" (Consejo de la UE, 2008: 2). Este documento establece también las directrices para los estados miembros tanto en objetivos a conseguir desde las políticas públicas, así como indicando la metodología y los instrumentos a utilizar para incrementar la presencia de la cultura en las actuaciones de acción exterior.

Tabla 1: Las bases de la dimensión exterior de la PAE.

\begin{tabular}{|l|l|}
\hline Año & Documento \\
\hline 2000 & Agenda de Lisboa \\
\hline 2005 & Convención UNESCO de la Diversidad Cultural \\
\hline 2006 & Comunicación “Europa en el mundo" \\
\hline 2007 & Agenda europea para la cultura en un mundo globalizado \\
\hline \multirow{2}{*}{2008} & Declaración de Cannes \\
\cline { 2 - 2 } & Conclusiones del Consejo Europeo \\
\hline \multirow{2}{*}{2009} & $\begin{array}{l}\text { Documento de trabajo de la Comisión sobre la dimensión exterior de } \\
\text { la PAE }\end{array}$ \\
\hline
\end{tabular}

Fuente: Elaboración propia.

Esta importancia creciente de la dimensión exterior dentro de la política audiovisual comunitaria se manifiesta una vez más con la presentación de un documento de trabajo de la Comisión Europea (2009c), bajo el título "La dimensión exterior de la política audiovisual" SEC (2009) 1033 final, publicado en julio de 2009 y que por primera vez abre a la luz pública las ideas de la Comisión sobre como se está construyendo la dimensión exterior de la PAE. En este documento se pone de relieve el protagonismo del bilateralismo frente al multilateralismo en las actuaciones comunitarias. En especial, se observa este cambio como consecuencia de la paralización de las negociaciones de la Ronda de Doha en el marco de la OMC, que ha llevado a la UE a impulsar nuevas técnicas para 
Audiovisual europeo, diversidad cultural y mercado global: Análisis de las actuaciones exteriores de la política audiovisual de la unión europea

conseguir aliados en la esfera internacional. En este sentido, la UE hace campaña y pide adhesiones a su modelo a otros países miembros de la OMC y a futuros candidatos. En este sentido, como afirma Peter Humphreys (2008: 78), “la UE no ha sido 'desreguladora' en relación a su política exterior. Más bien, la UE ha protegido sus políticas culturales intervencionistas y proteccionistasy de forma más relevante, considerando su capacidad limitada, las de sus estados miembros- contra las presiones estadounidenses para la liberalización de los servicios audiovisuales en las negociaciones internacionales de comercio". A pesar de protegerse de los EEUU, todo indica que el enfrentamiento euro- estadounidense vivido durante las negociaciones de la Ronda Uruguay ha desaparecido, porque los estadounidenses tienen otros frentes y están más preocupados por la piratería, en cuya lucha los europeos y los americanos colaboran intensivamente.

\section{EL MARCO DE ACTUACIÓN: LOS ASPECTOS LEGALES Y DE ORGANI- ZACIÓN}

El marco de actuación comunitario vendrá determinado por dos ejes fundamentales: el marco legal y la organización interna de la propia Comisión Europea. En el primero, habrá que tener en cuenta cuatro referencias principales:

1. El artículo 151 del Tratado de la UE que expresa que la Unión puede fomentar con terceros países y con las organizaciones internacionales competentes en temas culturales.

2. La Convención sobre la Protección y Promoción de la Diversidad de las Expresiones Culturales de la UNESCO: en especial, los artículos 20, que requiere tener en cuenta esta convención en todos los acuerdos que se realicen; el 16, que otorga tratamiento preferencial a los artistas y a los profesionales culturales y los practicantes de los países en desarrollo; y el 12 que precisa fortalecer la cooperación bilateral, regional e internacional para la creación de condiciones propicias a la promoción de la diversidad de las expresiones culturales.

3. La Directiva de Servicios de Comunicación Audiovisual (DSCA) ha adaptado la definición de "obra europea" de tal manera que permite que las obras co-producidas en el marco de acuerdos relativos al sector audiovisual, concluidos entre la Comunidad y terceras partes, y bajo esas condiciones, serán tratados como "europeas" para los propósitos de los requerimientos de la directiva en la promoción de obras audiovisuales, tanto en los medios lineales como en los no-lineales (Parlamento Europeo y Consejo de la UE, 2007).

4. El programa MEDIA 2007 también ha adaptado su articulado para la cooperación con terceros países (artículo 8) (Parlamento Europeo y Consejo de la UE, 2006). 
En relación a la organización interna de las instituciones comunitarias, en concreto de la Comisión Europea, bajo el segundo mandato de la Comisaria Viviane Reding (2004-2009), como muestra la tabla 2, la acción exterior de la PAE se ha dividido en cinco áreas: ampliación, política de vecindad, relaciones comerciales (OMC y OCDE), promoción de la diversidad cultural y cooperación internacional.

Tabla 2: Áreas de la acción exterior de la PAE.

\begin{tabular}{|c|c|c|c|c|}
\hline Ampliación & $\begin{array}{c}\text { Política de } \\
\text { vecindad }\end{array}$ & $\begin{array}{c}\text { Relaciones } \\
\text { comerciales }\end{array}$ & $\begin{array}{c}\text { Promoción } \\
\text { Diversidad } \\
\text { Cultural }\end{array}$ & $\begin{array}{c}\text { Cooperación } \\
\text { Internacional }\end{array}$ \\
\hline $\begin{array}{c}\text { Países } \\
\text { candidatos } \\
\text { Países } \\
\text { potenciales }\end{array}$ & $\begin{array}{c}\text { Países del } \\
\text { Mediterráneo }\end{array}$ & OMC & $\begin{array}{c}\text { Implementación } \\
\text { OC la Convención } \\
\text { UNESCO }\end{array}$ & $\begin{array}{c}\text { Europea del Este } \\
\text { Mediterráneo } \\
\text { Cáucaso } \\
\text { América Latina } \\
\text { (MERCOSUR) }\end{array}$ \\
\hline
\end{tabular}

Fuente: Elaboración propia a partir de los datos de la web oficial de la Comisión Europea (http://ec.europa.eu/avpolicy/ext/index en.htm).

Será a partir de estas áreas que la PAE desarrolla su dimensión exterior, pero lo importante es destacar que en los dos últimos años se ha dado importancia a la dimensión más global. Es decir, que existe una firme voluntad por parte de la Unión de estar presente en el nuevo escenario mundial y priorizar las acciones de carácter internacional que colaboran en posicionar a la UE como una de los actores clave en el mundo.

La experiencia para ampliar el ámbito de acción de la PAE se ha ido adquiriendo con la propia ampliación interna de la UE, especialmente con la incorporación de 10 países en el 2004. Así, en este proceso se establecen los requisitos mínimos para formar parte de la Comunidad en el ámbito audiovisual. Estos se concretan en lo que se denomina como los "estándares europeos", que equivalen a dos: primero, asegurar que los medios de comunicación funcionan de acuerdo con los principios democráticos fundamentos de los llamados "criterios de Copenhaguen"; y segundo, sus leyes deben estar en línea con la Directiva de Servicios de Comunicación Audiovisual (DSCA).

Se ha completado, por otra pare, la experiencia comunitaria con la zona geográfica más próxima, bajo el dominio de la política de vecindad, especialmente centrada en los países del Mediterráneo, donde se ha desarrollado un partenariado cultural euro-mediterráneo, que se incluye en un programa de cooperación 
Audiovisual europeo, diversidad cultural y mercado global: Análisis de las actuaciones exteriores de la política audiovisual de la unión europea

cultural. En concreto, existe uno para el sector audiovisual, llamado Euromed Audiovisual $^{2}$.

En las relaciones comerciales, con marco principalmente en la OMC y OCDE, en especial en el primer organismo, la UE ha tratado de promocionar su modelo de no adhesión a la cláusula de nación más favorecida para el audiovisual entre los países miembros y los nuevos candidatos. Este hecho ha puesto de manifiesto que a pesar de ser un entorno multilateral, la estrategia de la UE se basa en el bilateralismo para conseguir crear un pool de países que apoyen la posición europea.

En el caso de la Diversidad Cultural, la UE se ha erigido en el gran promotor de esta convención a nivel mundial. A pesar de estar presente en todos los documentos como el motivo de toda actuación internacional, esta defensa de la diversidad cultural no es ajena a un debate sobre si su inclusión en las actuaciones comunitarias es una mera fórmula retórica o realmente se trata de una verdadera voluntad de defensa del concepto. Voces autorizadas, con las que estamos de acuerdo, señalan que de momento existen todavía ciertos elementos de conflicto que dificultan la definición y aplicación completa. Como señalan De Vinck \& Pauwels (2008: 302-303), sigue existiendo una lucha entre los Estados miembros y la UE sobre las competencias en el ámbito de la cultura, a pesar de la existencia del artículo 151, incorporado por el Tratado de Maastricht, y las fronteras de la subsidiariedad no siempre son claras. Asimismo, como afirman estas autoras, "a pesar de este discurso, en la práctica el tema de la diversidad cultural se refiere mayormente a las competencias a nivel de estado miembro", además "muchas de las definiciones y las reglas relevantes adoptadas a nivel europeo son sumamente difusas, por lo que su aplicación desde el principio es difícil". En definitiva, "bastante paradójicamente, parece que la principal amenaza a las preocupaciones sobre la diversidad cultural actualmente emana de la imprecisión y ambigüedad de las disposiciones relevantes de la UE” (2008:304). La única solución, según estas autoras, se encontraría en que "los Estados miembros demostrarán su voluntad política para trascender de la diversidad nacional a la diversidad europea y nacional". Es decir, el voluntarismo de las instituciones europeas debería trasladarse a los Estados miembros, y de esta manera, se conseguiría un gran avance para poder pasar a una defensa real de la diversidad cultural.

Por último, en el área dedicada a la cooperación internacional, ha ido adquiriendo protagonismo en su vertiente más técnica, y ha tenido al MERCOSUR como el principal protagonismo. Se cree que tras esta experiencia, se podría desarrollar en nuevas ocasiones con países asiáticos, que están siendo una de las prioridades exteriores de la UE.

EI EUROMED AUDIOVISUAL II (2006-2009), con una dotación total de 15 millones de euros, se centra en la conservación de archivos, el fomento de la producción y la distribución de obras audiovisuales. 
Estas áreas de actuación de la Comisión Europea (2009c) se han visto complementadas por las directrices que plantea el documento de la posición europea SEC (2008) 3097 final (9/1/09), en el que se especifica cuál debe ser el rol de Europa en el mundo, con el objetivo de aumentar su credibilidad; una comparativa con Estados Unidos; y se señala la coproducción como instrumento básico para desarrollar la cooperación internacional.

\section{LOS INSTRUMENTOS DE LA ACCIÓN EXTERIOR}

La puesta en marcha de esta dimensión exterior de carácter más global ha supuesto la utilización de determinados conceptos e instrumentos. Sobre los primeros, el concepto central es la cooperación bajo dos prismas: el primero, la perspectiva del desarrollo se utiliza con países en vías de desarrollo, como se hizo en el caso de CARIFORUM, para crear medidas de apoyo a la industria audiovisual. El segundo, el del intercambio equilibrado, o mejor dicho, la cooperación con beneficio mutuo, que se entiende más en el sentido económico, bajo un paraguas de protección cultural, como ha sido el caso de Corea.

En todos los casos, el marco de la acción comunitaria tendrá cuatro objetivos (Comisión Europea, 2007): implementar la Convención de la UNESCO; animar a los socios con los que se negocia a ratificar la Convención UNESCO rápidamente, si todavía no lo han hecho; reconocer la importancia de las industrias culturales y la mutidimensión de los bienes y servicios culturales como actividades de valor cultural, económica y social y facilitar el intercambio entre bienes y servicios culturales; y asegurar que el sector audiovisual es negociado dentro de las provisiones de la cooperación dentro de un título específico de los acuerdos comerciales de la UE y por lo tanto no estará cubierto por las disposiciones comerciales en servicios.

Los resultados que se esperan tienen triple vertiente: un incremento de los intercambios en bienes y servicios culturales, dando especial tratamiento a las coproducciones con los países en desarrollo; la promoción internacional y estándares europeos en el sector audiovisual; y facilitar la entrada en territorios de los socios de profesionales y trabajadores culturales extranjeros para actividades que no constituyen disposición de servicios. Las autoridades comunitarias han marcado como áreas prioritarias de actuación (Comisión Europea, 2007 y 2008d): ASEAN, Comunidad Andina y América Central, los países Euromed, Corea, India, y África Central.

Ante estos objetivos y resultados esperados, podemos situar la acción comunitaria con una doble dinámica, como nos muestra la tabla 3: la cultural, con la implementación y promoción de la Convención de la Diversidad Cultural; y la económica, con la búsqueda de nuevos mercados para las obras europeas. Para conseguir ambos propósitos, durante el periodo de análisis de este articulo, se han estudiado tres tipos de instrumentos utilizados por las instituciones comunitarias. El primero, los protocolos de cooperación cultural, que acompañan a los acuerdos 
Audiovisual europeo, diversidad cultural y mercado global: Análisis de las actuaciones exteriores de la política audiovisual de la unión europea

comerciales entre la UE y otros países y/o regiones. El segundo, la creación de un programa de ámbito internacional de apoyo a la industria audiovisual en el marco del programa MEDIA, conocido como MEDIA INTERNACIONAL y MEDIA MUNDUS. El tercero, utiliza la cooperación técnica para ayudar a terceros a definir su política audiovisual.

Tabla 3: Los instrumentos de la acción exterior de la PAE.

\begin{tabular}{|c|c|c|}
\hline \multicolumn{3}{|c|}{ Dinámica cultural: } \\
CONVENCIÓN DE LA DIVERSIDAD CULTURAL \\
\hline $\begin{array}{c}\text { INSTRUMENTO 1 } \\
\text { Protocolo de cooperación } \\
\text { cultural }\end{array}$ & $\begin{array}{c}\text { MEDIA INTERNATIONAL } \\
\text { MEDIA MUNDUS }\end{array}$ & COOPERACIÓN TÉCNICA \\
\hline \multicolumn{3}{|c|}{ INSTRUMENTO 3 } \\
MERĆmica económica: \\
MERO AUDIOVISUAL GLOBAL \\
\hline
\end{tabular}

Fuente: Elaboración propia.

\subsection{Instrumento 1: Protocolos de cooperación cultural}

El primer instrumento se enmarca en la cooperación cultural como complemento a los acuerdos comerciales firmados por la UE y otros países, a través de la definición de protocolos anexos ${ }^{3}$. El protocolo cultural sirve de instrumento para implementar la Directiva de Servicios de Comunicación Audiovisual (DSCA), que provee reforzar la diversidad cultural a través de la extensión de la definición de las obras europeas a ciertas coproducciones audiovisuales con terceros países bajo específicas condiciones establecidas en cada caso.

Hasta finales de 2009, se han firmado dos protocolos de cooperación cultural. El primero fue el Protocolo de Cooperación Cultural del Acuerdo de Asociación Económica firmado con catorce países del grupo CARIFORUM en octubre de 2008 (Parlamento Europeo y Consejo de la UE, 2008). El segundo con Corea del Sur en octubre de 2009. Este último tuvo algunos reticencias iniciales por la negativa de algunos Estados miembros, así como de organizaciones profesionales como la Federación Europea de Realizadores Audiovisuales (FERA), a firmarlo (Comisión Europea, 2009d y 2009e). De los protocolos ya firmados, destacan algunas características. En el caso del CARIFORUM, la base se encuentra en los acuerdos de coproducción dentro del maco de la DSCA. Se establece un

\footnotetext{
${ }^{3}$ Sobre esta temática, es interesante consultar Formentini e lapadre (2007).
} 
compromiso por ambas partes del uso de los estándares regionales e internacionales para asegurar la compatibilidad e interoperabilidad de las tecnologías audiovisuales, contribuyendo de esta manera a fortalecer los intercambios culturales. Se incluyen condiciones especiales para la importación temporal de material y equipamiento con el propósito del rodaje de obras audiovisuales. En el protocolo firmado con Corea del Sur, la base ya no es cooperación al desarrollo, sino una lógica de estricta reciprocidad y equilibrio. Se parte de la consideración que existe una situación similar entre las industrias audiovisuales (una política pública de ayuda y promoción a la industria, así como la existencia de una política de cuotas). Además, se establece la coproducción como instrumento de introducción de los productos europeos en otros mercados, tan crecientes como el coreano y en su extensión, el asiático. Se detallan las condiciones para asegurar la estricta equivalencia. El protocolo final en cooperación cultural incluye una sub-sección A con disposiciones relativas a las obras audiovisuales, detallando las características de las coproducciones para que se puedan beneficiar de los programas de apoyo y fomento de la industria de ambos países. También se incluye otro tipo de cooperación audiovisual (organización de festivales, sector de la radiodifusión, establecimientos de estándares comunes para asegurar la compatibilidad e interoperabilidad de las tecnologías audiovisuales) y condiciones especiales para la importación temporal de material y equipamiento con el propósito del rodaje de obras audiovisuales. En definitiva, nos encontramos ante un ejemplo de cooperación con beneficio mutuo para promocionar la circulación de las obras europeas en mercados emergentes (Gobierno de Corea, 2009).

\subsection{Instrumento 2: MEDIA INTERNATIONAL y MEDIA MUNDUS}

El segundo instrumento se enmarca dentro de uno de los pilares de la PAE: el programa MEDIA. Durante el mandato de la Comisión Barroso I (2004-2009) se ha presentado una novedad: su ampliación en el ámbito internacional, extendiendo las fronteras del programa hacia el entorno global. Son los primeros pasos que muestran una cierta voluntad de exportar el modelo europeo y un claro ejemplo de la importancia creciente de la dimensión exterior de la política audiovisual. En concreto se han realizado dos acciones: una preparatoria, denominada MEDIA INTERNATIONAL (Comisión Europea, 2008a, 2009a y 2009b), y una ampliación del programa, MEDIA MUNDUS (Comisión Europea, 2008b, 2009f, 2009 g y 2009 h).

La primera acción preparatoria, MEDIA Internacional, ha tenido como objetivo explorar las maneras de reforzar la cooperación entre la Unión Europea y los profesionales audiovisuales no europeos. Este programa espera funcionar durante tres ediciones $(2008,2009,2010)$ para preparar el camino al nuevo programa MEDIA MUNDUS, que se activará a partir del 2011 y durará hasta el 2013. Es una muestra clara de la voluntad de la Comisión Europea de dar mayor protagonismo a la dimensión exterior, sobre todo teniendo en cuenta que en el mercado global el audiovisual europeo puede encontrar nuevos aliados y otras vías de comercialización. 
Audiovisual europeo, diversidad cultural y mercado global: Análisis de las actuaciones exteriores de la política audiovisual de la unión europea

Tabla 4: Resultados de las dos convocatorias del programa MEDIA INTERNATIONAL.

\begin{tabular}{|c|c|c|c|}
\hline \multicolumn{2}{|c|}{$1^{\text {a }}$ Convocatoria (2008) } & \multicolumn{2}{|c|}{$2^{\text {a }}$ Convocatoria (2009) } \\
\hline \multicolumn{2}{|c|}{$€ 2$ millones } & \multicolumn{2}{|c|}{$€ 5$ millones } \\
\hline 33 proyectos & 18 seleccionados & 100 proyectos & 40 seleccionados \\
\hline $\begin{array}{l}\text { № proyectos } \\
\text { seleccionados } \\
\text { según temática }\end{array}$ & Áreas geográficas & $\begin{array}{l}\text { № proyectos } \\
\text { seleccionados } \\
\text { según temática }\end{array}$ & Áreas geográficas \\
\hline $\begin{array}{l}11 \text { - Formación } \\
6 \text { - promoción y } \\
\text { distribución } \\
1 \text {-apoyo red in- } \\
\text { ternacional exhi- } \\
\text { bición }\end{array}$ & $\begin{array}{l}\text { América Latina: paí- } \\
\text { ses MERCOSUR } \\
\text { Asia: Corea, India, } \\
\text { Japón } \\
\text { Otros: Turquía, Mol- } \\
\text { davia, Ucrania, Ca- } \\
\text { nadá }\end{array}$ & $\begin{array}{l}23 \text { - formación } \\
10 \text { - acceso merca- } \\
\text { do internacional } \\
4 \text { - distribución } \\
1 \text { - apoyo red inter- } \\
\text { nacional exhibición } \\
2 \text { - aumentar público } \\
\text { potencial }\end{array}$ & $\begin{array}{l}\text { América Latina: MERCO- } \\
\text { SUR, México, Costa Rica, } \\
\text { Colombia, Ecuador y Perú } \\
\text { Asia: Japón, Corea, India, } \\
\text { Hong Kong, China, Singa- } \\
\text { pur, Malasia } \\
\text { Europa: Bosnia, Kosovo, } \\
\text { Macedonia, Turquía } \\
\text { Oriente Medio: Dubái, Is- } \\
\text { rael, Siria } \\
\text { África: Nigeria, Egipto, Ar- } \\
\text { gelia, Marruecos y Túnez } \\
\text { Otros: Australia, EEUU, } \\
\text { Rusia, Canadá, Ucrania y } \\
\text { Armenia. }\end{array}$ \\
\hline
\end{tabular}

Fuente: Elaboración propia a partir de los datos de la Comisión Europea (2009a, 2009b, 2008a).

Como nos muestra la tabla 4, las primeras dos convocatorias (2008 y 2009) ponen de relieve el interés creciente por el programa que no sólo ha ampliado sus áreas de actuación, sino también la participación de una mayor diversidad de países. A modo de ejemplo, la acción preparatoria del MEDIA Internacional provee financiación a proyectos en los que participen terceros países, entre ellos los países miembros de América Latina (en especial, con el MERCOSUR). De los proyectos seleccionados en la primera edición (2008), cinco incluyen participantes de Argentina, Brasil, Chile y Uruguay. La financiación asciende a €346.480. Existe también un proyecto de crear una red de cines que agrupa a 23 cines en países del MERCOSUR. Los resultados de esta acción han sido muy difundidos en la región sudamericana como un gran éxito para sus empresas audiovisuales. De la segunda edición (2009), diez proyectos incluyen participantes del MERCOSUR, con una ayuda total de $€ 1.238$.116. De la red de cines patrocinada por Europa Cinemas Internacional, se incluyen 247 salas latinoamericanas que recibirán ayudas. 
Siguiendo con esta idea de ampliación global, a principios de 2009 se presentó el nuevo programa MEDIA MUNDUS, con un presupuesto de 15 millones de euros (2011-2013), para la cooperación con profesionales audiovisuales de terceros países. Sus objetivos se centran principalmente en mejorar: a) el intercambio de información entre profesionales europeos y de terceros países; b) la competitividad y la distribución transnacional de las obras europeas; c) la circulación y exposición de las obras europeas a nivel mundial; y d) incrementar la demanda del público en contenido audiovisual culturalmente diverso (Parlamento Europeo, 2009: 288-312). El programa MEDIA MUNDUS se concibe como una iniciativa para incrementar la competitividad del sector audiovisual en el mercado global y contribuir a la implementación de la Convención de la UNESCO sobre la promoción y protección de la diversidad de las expresiones culturales. También se apunta la posibilidad de incrementar las opciones del consumidor y la diversidad cultural en los mercados europeos. Hay que tener en cuenta un aspecto mencionado en el estudio preliminar: las obras audiovisuales europeas tienen dificultades en el mercado internacional y consiguen una circulación muy pobre. Por eso el MEDIA MUNDUS puede suponer facilitar la entrada de otros mercados. Esta oportunidad no se ha querido desaprovechar por parte de las instancias comunitarias. Un reflejo de esta trascendencia está en su proceso de aprobación por parte del Consejo y del Parlamento Europeo que ha sido realmente rápido, quedando aprobado el programa bajo la decisión 1041/2009/EC el 21 de octubre de 2009, en menos de un año.

\subsection{Instrumento 3: La cooperación internacional técnica}

El tercer instrumento de la acción exterior es la cooperación técnica entre la UE y otra región. En este caso, el ejemplo más ilustrativo es el caso del MERCOSUR. La cooperación entre el MERCOSUR y la Unión Europea ha sido uno de los ejes de actuación que se marcó desde los inicios las autoridades audiovisuales del MERCOSUR, agrupadas en la RECAM (Reunión Especializada de Autoridades Cinematográficas y Audiovisuales del MERCOSUR). Esto es, se busca en el modelo europeo una referencia para diseñar algunos aspectos de la política audiovisual del MERCOSUR, y al mismo tiempo una posible fuente de financiación para algunas de sus actuaciones. La misma institución afirma que "la amplia experiencia de la Unión Europea en materia audiovisual, en particular del programa Media, ha sido un insumo fundamental para la elaboración de lineamientos de la RECAM". En el año 2004 se elaboró una propuesta para la cooperación técnica con la UE en materia cinematográfica y audiovisual relacionada con la preservación del patrimonio audiovisual, los sistemas de información y programas, y las políticas cinematográficas y audiovisuales. Posteriormente se procedió a presentar el proyecto al Comité de Cooperación Técnica, cuyos resultados se pudieron observar cuando en febrero de 2007 cuando se incluye al sector cinematográfico y audiovisual como sector prioritario 3 para la cooperación UE-MERCOSUR para el periodo 2007-2013 (RECAM, 2008). 
Audiovisual europeo, diversidad cultural y mercado global: Análisis de las actuaciones exteriores de la política audiovisual de la unión europea

Es importante también ubicar esta cooperación en el marco general de las relaciones entre las dos entidades supranacionales UE-MERCOSUR. En concreto, las acciones vinculadas al sector audiovisual se enmarcarán dentro de las actividades prioritarias relacionadas con los temas de integración y su relación con la sociedad civil. La base de la actuación está en considerar que la cooperación en el área audiovisual puede contribuir a aproximar el proceso de integración a los ciudadanos.

Desde la vertiente europea, la cooperación UE-MERCOSUR tendrá dos protagonistas: el Parlamento Europeo y la Comisión Europea. Ambas instituciones participan en acciones con objetivos diferentes y a su vez complementarios. En el primer caso, se enmarcan en las acciones de cooperación con países de ingresos medios en América Latina; y en el segundo, se sitúan dentro de las acciones en apoyo de la industria audiovisual, a través del programa MEDIA MUNDUS.

La cooperación liderada desde el Parlamento Europeo está vinculada a la acción preparatoria - "Cooperación con los países de ingresos medios en América Latina" (con la línea de presupuesto 1909 02) creada en 2008, cuyas provisiones serían usadas para dar apoyo a acciones que estuvieran fueran del alcance del instrumento de regulación de cooperación al desarrollo. Dentro de este ámbito se encuentra el programa MERCOSUR Audiovisual, en línea con el informe sobre la estrategia regional del MERCOSUR (2007-2013), adoptado por la Comisión Europea el 23 de diciembre de 2008, con un presupuesto de 1'5 millones de euros para el 2008 y cuya implementación está prevista hasta mediados de 2009 (COMISIÓN EUROPEA, 2009c: 22-23).

El objetivo general del programa es fortalecer al sector cinematográfico y audiovisual en el MERCOSUR como un instrumento para fomentar el proceso de integración regional y la participación de la sociedad civil. El Programa está diseñado para mejorar el acceso de los ciudadanos a los contenidos propios por medio de una serie de acciones coordinadas para promover la complementación de las industrias cinematográficas, facilitar la circulación intrarregional y fortalecer las capacidades técnicas reduciendo simetrías ${ }^{4}$.

Las actividades del programa serán desarrolladas a partir de cinco ejes de intervención:

1) Eje 1: Elaborar estudios comparativos para la armonización de la legislación en el sector audiovisual en los países del MERCOSUR.

2) Eje 2: Fortalecer las capacidades del Observatorio MERCOSUR Audiovisual para generar y suministrar información específica para el diseño de políticas públicas audiovisuales regionales.

\footnotetext{
${ }^{4}$ El problema central del MERCOSUR Audiovisual es la poca presencia de contenidos propios en las distintas y diversas ventanas de exhibición, lo que no favorece la construcción de una ciudadanía regional. Ello se debe, principalmente, a la escasa circulación de contenidos propios a nivel intra-regional, a la falta de estrategias nacionales y regionales para los nuevos modelos de comercialización, lo que implica la convergencia digital, y a las asimetrías en la producción de contenidos audiovisuales.
} 
3) Eje 3: Implementar una red de 30 salas digitales en los países del MERCOSUR donde circularán los contenidos audiovisuales propios de la región.

4) Eje 4: Difundir el patrimonio audiovisual del MERCOSUR restaurado, conservado y digitalizado.

5) Eje 5: Fortalecer las capacidades técnicas (tecnológicas, comerciales y artísticas) del sector audiovisual, con especial foco en Paraguay.

Estas cinco líneas de acción son totalmente complementarias a las actuaciones planteadas por la Política Audiovisual del MERCOSUR, y en algunos casos, la cooperación europea se convierte en la vía de financiación que se estaba buscando.

\section{CONCLUSIONES}

El análisis de la dimensión exterior nos va proporcionando los rasgos de lo que podríamos llamar el modelo europeo, base de la actuación global de la UE. Bajo un enfoque cultural basado en la Convención UNESCO de la Diversidad Cultural, se utilizan dos estrategias de actuación: la perspectiva de cooperación al desarrollo y la de cooperación con beneficio mutuo. Los tres instrumentos utilizados ponen de relieve que la dimensión económica no pierde protagonismo; al contrario, está presente en cada una de las acciones comunitarias, poniendo de relieve que el mandato de mercado sigue siendo predominante.

La actuación comunitaria se caracteriza por ser de naturaleza eminentemente bilateral bajo el paraguas de escenarios multilaterales, siendo la UNESCO y la $\mathrm{OMC}$ las que marcan las reglas del juego global. En este nuevo escenario, el objetivo es conseguir situar a la UE como un player central en el nuevo escenario global, acompañado de un conjunto de países socios y áreas regionales. EI bilateralismo se convierte en el camino indispensable para conseguir reforzar el posicionamiento en el nuevo multilateralismo global.

Esta presencia creciente de la dimensión exterior es sólo un avance de la importancia que puede tomar en los próximos años cuando el Tratado de Lisboa se ponga en práctica completamente. Esto es, cuando la alta representante, Catherine Ashton, quien según el artículo 9E tendrá que velar por la coherencia en la acción exterior de la Unión, despliegue toda la acción exterior de la UE y realmente consiga situar a la UE como un actor relevante y decisivo en el escenario mundial.

En este escenario emerge lo que se denomina la exportación del modelo europeo audiovisual. Éste se puede definir a partir de las acciones de la UE en los últimos años, que serían el resultado de sumar cuatro dimensiones. La dimensión económica tendrá protagonismo en tanto que búsqueda de nuevos mercados para las obras europeas y conseguir nuevas alianzas para la coproducción. Tendría sus aliados en los protocolos de cooperación cultural y los programas MEDIA INTERNATIONAL y MEDIA MUNDUS. La dimensión cultural tendrá una presencia 
Audiovisual europeo, diversidad cultural y mercado global: Análisis de las actuaciones exteriores de la política audiovisual de la unión europea

dominante en el discurso de la UE, bajo la promoción de la Convención de la Diversidad Cultural. La parte política, o mejor dicho geopolítica, la encontramos en las actuaciones de la UE en los escenarios de la OMC y de la UNESCO, buscando alianzas bilaterales para posicionar a la UE frente a los EEUU. Existe también otra dimensión, que sería objeto de un análisis particular, que seria la tecnológica. Esto es, la exportación del estándar DVB de televisión digital, que está siendo adoptado por otros países no europeos. Todas estas dimensiones configuran lo que podríamos llamar el modelo europeo en su dimensión exterior.

Esta acción exterior de la PAE no está exenta de críticas por parte de otros actores internacionales. Muchos acusan a la UE de primar el mandato de mercado y es acusada de un enfoque agresivo hacia la liberalización de los bienes y servicios y de falta de transparencia en las negociaciones. Otros señalan las contradicciones entre el enfoque región-región y el enfoque bilateral en las negociaciones. Buen ejemplo de ello son los fracasos en las propuestas de acuerdos de libre comercio con la ASEAN (se encuentra en una etapa de no avance) y el fracaso con la Comunidad Andina, que ha desembocado en el inicio de las negociaciones para los acuerdos bilaterales con Colombia, Perú y Ecuador.

En definitiva, nos encontramos ante un tema relevante que recién toma protagonismo y que será necesario seguir analizando para realmente afirmar la existencia de un modelo europeo único y definido que está siendo exportado, ya que hasta estos momentos sólo empiezan a aparecer indicios y la cuestión de futuro será analizar si realmente este modelo europeo desarrolla modelos de cooperación o por el contrario, crea nuevas dependencias con otros países o regiones.

\section{REFERENCIAS BIBLIOGRÁFICAS}

BURRI-NENOVA, M. (2007): "The New Audiovisual Media Services Directive: Television without Frontiers, television without diversity”, Common Market Law Review, vol. 44 (2007), $\mathrm{n}^{\circ} 6$, pp. 1689-1725.

BUSTAMANTE, E.: Public Service in the Digital Age: Opportunities and Threats in a Diverse Europe. En FERNÁNDEZ ALONSO, I.; y MORAGAS, M. (eds.) (2008): Communication and Cultural Policies in Europe. Bellaterra: Universidad Autónoma de Barcelona, pp. 185-216.

CHAKRAVARTTY, P. y SARIKAKIS, K. (2006): Media Policy and Globalization. Edinburgh: Edinburgh University Press.

COMISIÓN EUROPEA (2009a): El cine europeo se mundializa: 40 proyectos internacionales de cooperación cinematográfica reciben ayudas comunitarias por un importe de 5 millones de euros, IP/09/1334, 18 de septiembre. [Consulta: 2 diciembre 2009]. http://europa.eu/ra$\mathrm{pid} /$ pressReleasesAction. do? reference $=\mid \mathrm{P} / 09 / 1334 \&$ format $=\mathrm{HTML} \&$ aged $=0$ \&language $=\mathrm{EN}$

- (2009b): Results of 2nd call MEDIA INTERNATIONAL Preparatory Action 2009, septiembre. [Consulta: 2 diciembre 2009].

http://ec.europa.eu/information_society/media/docs/international/projects/list2009.pdf 
- (2009c): External Dimension of Audiovisual Policy (Staff Working Document), SEC (2009) 1033 final, 14/7/2009. [Consulta: 30 julio 2009].

http://ec.europa.eu/avpolicy/docs/ext/external_swp.pdf

- (2009d): Concept Paper: Cultural Cooperation with Korea, 18 febrero. [Consulta: 20 junio 2009].

http://trade.ec.europa.eu/doclib/docs/2009/march/tradoc_142542.pdf

- (2009e): Background Protocol on Cultural Cooperation with Korea, 26 de enero. [Consulta: 20 junio 2009].

http://trade.ec.europa.eu/doclib/docs/2009/march/tradoc_142541.pdf

- (2009f): Proposal for a Decision Of The European Parliament And Of The Council establishing an audiovisual cooperation programme with professionals from third countries MEDIA Mundus (COM(2008) 892 final), 9 de enero. [Consulta: 1 septiembre 2009].

http://ec.europa.eu/information_society/media/docs/mundus/mundus_proposal_en.pdf

- (2009g): Media Mundus. Summary of the Impact Assessment, SEC (2008) 3097 final, 9/1/09, Bruselas. [Consulta: 20 junio 2009].

http://ec.europa.eu/information_society/media/docs/mundus $/ \mathrm{mm}$ ria/en.pdf

- (2009h): Cinema: EU film support goes global, IP/09/26, 9 enero. [Consulta: 20 junio 2009].

http://europa.eu/rapid/pressReleasesAction.do?reference=IP/09/26\&format=HTML\&aged= $\underline{0 \text { \&language }=E N \& g u i L a n g u a g e}=e n$

- (2008a) EU Film support programme's first global trigger interest in Asia and South America, IP/08/1273, 27 de agosto.

- (2008b): Audiovisual Cooperation Programme "MEDIA MUNDUS". Summary of the results of the public consultation [Consulta: 20 junio 2009].

http://ec.europa.eu/information_society/media/docs/mundus/pc.pdf

- (2008c) Cannes Declaration / Declaration of European Audiovisual Affairs Ministers and of the European Commission member responsible for the Information Society and Media, Europe Day at the Cannes Festival, 19 de mayo. [Consulta: 20 junio 2009].

http://ec.europa.eu/avpolicy/docs/ext/cannes_2008 en.pdf

- (2008d) Follow-up Argumentaire On the Cultural Cooperation Protocol in future EU trade Agreements, 13 de febrero. [Consulta: 20 junio 2009].

http://trade.ec.europa.eu/doclib/docs/2008/february/tradoc_137751.pdf

- (2007) Argumentaire On the Cultural Cooperation Protocol in future EU trade Agreements, mayo. [Consulta: 21 junio 2009].

http://www.efah.org/components/docs/argumentaire\%20EN.pdf

CONSEJO DE LA UNIÓN EUROPEA (2008): Council Conclusions on the promotion of cultural diversity and intercultural dialogue in the external relations of the Union and its Member States, 2905th Education, Youth and Culture Council meeting, 20 noviembre. [Consulta: 20 junio 2009].

http://ec.europa.eu/culture/ourpolicydevelopment/doc/ICD external relations en.doc.pdf 
Audiovisual europeo, diversidad cultural y mercado global: Análisis de las actuaciones exteriores de la política audiovisual de la unión europea

- (2007): Draft Council Resolution on a European agenda for culture - Adoption of the Resolution, 14485/07, 31 de octubre. [Consulta: 20 junio 2009].

http://www.cultureactioneurope.org/images/stories/advocatepdf/eucouncilresolutioneuagend aforculture31.10.07en.pdf?e4b73c3745ac4bc374714928e835769b=163d48973a71d05dc42 ea8e8bfeae4fc

CRUSAFON, C.: "Mercado vs Cultura: balance de la Comisión Barroso (2004-2009)", Estudos em Comunicaçao, no 6 (diciembre 2009), pp.105-123.

- (2009b): "La política audiovisual del MERCOSUR y la influencia del modelo europeo", Cuadernos de Información, no 25 (diciembre 2009), pp. 93-104.

- El espacio audiovisual euro-latinoamericano: análisis de los programas IBERMEDIA y MEDIA como motores de la integración cinematográfica. En AA.VV. (2009): Actas XI IBERCOM, Funchal, 16-19 de abril. Funchal: Universidade da Madeira [en prensa].

- Los desafíos de la política audiovisual europea en la Europa de los 25. En AA.VV. (2005): Actas del III Congreso Panamericano, Buenos Aires, 12-16 de julio. Buenos Aires: Universidad de Buenos Aires, CD-Rom.

- ¿Qué futuro para la política audiovisual europea? Análisis y resultado de la aplicación de una política supranacional en la industria audiovisual. En AA.VV. (2001): Actas VIII Jornadas Internacionales de la Asociación Internacional de Jóvenes Investigadores en Comunicación. Universidad de La Plata (Argentina), 27-28 de agosto de 2001. Argentina: Universidad de La Plata, CD Rom.

- (1999): El espacio audiovisual europeo. Análisis de la industria audiovisual y de las políticas europeas en la década de los 90. Tesis doctoral inédita, Universidad Autónoma de Barcelona.

DE COCKKBORNE, J.E. y TRETTENBREIN, H.: European Media Governance and the Role of the European Comission. En TERZIS, G. (ed.) (2008): European Media governance. The Brussels Dimension. Bristol: Intellect, pp. 27-42.

DE VINCK, S. \& PAUWELS, C.: Cultural diversity as the final outcome of EU policymaking in the audiovisual sector: a critical analysis. En SCHNEIDER $H$. y VAN DEN BOSSCHE P. (eds.) (2008): Protection of Cultural Diversity from a European and International Perspective. Antwerpen/Oxford/Portland: Intersentia, pp. 263-316.

DE VINCK, S., BILTEREYST, D. \& PAUWELS, C.: Our Policies Keep on Reinventing the Past: An Overview of EU Policy-Making in the Audiovisual Domain. En D'HAENENS, L. y SAEYS, F. (eds.) (2007): Western Broadcast Models. Structure, Conduct and Performance. Berlin: Mouton de Gruyter, pp. 25-59.

FOUCHER, M. (2009): “Quelles frontiers pour l'Europe?”, Le Monde, 13 octubre, pág.24.

FLEW, T. (2007): Understanding Global Media. New York: Palgrave.

FRAU-MEIGS, D.: The right to Cultural Diversity: UNESCO's Role in the International Debate on Circulation of Cultural Gods and Services and their Influence on Cultural and Media Policies. En FERNÁNDEZ ALONSO, I.; y MORAGAS, M. (eds.) (2008): Communication and Cultural Policies in Europe. Bellaterra: Universidad Autónoma de Barcelona, pp. 84-121. 
FORMENTINI, S.; y IAPADRE, L. (2007): "Cultural Diversity and Regional Trade Agreements: The Case of Audiovisual Services", UNU - CRIS Working Papers, $n^{\circ} 4$ (winter 2007), pp.1-25.

FREEDMAN, D. (2008): The Politics of Media Policy. Cambridge (UK): Polity.

GALPERÍN, H.: "Cultural Industries Policy in Regional Trade Agreements: the cases of NAFTA, the European Union and MERCOSUR", Media, Culture \& Society, n 21 (1999), pp.627-648.

GOBIERNO DE COREA DEL SUR (2009): Protocol on Cultural Cooperation, octubre. [Consulta: 15 diciembre 2009].

http://www.fta.go.kr/pds/fta_korea/eu/eu_proto3 e.pdf

GUERRIERI, P., IAPADRE L. y KOOPMANN, G. (eds.). (2005): Cultural Diversity and International Economic Integration. The Global Governance of the Audio-Visual Sector. Cheltenham (UK): Edward Elgar.

HARCOURT, A. (2008): Introduction. En TERZIS, G. (ed.): European Media governance. The Brussels Dimension. Bristol: Intellect, pp.13-23.

- (2005): The European Union and the regulation of media markets. Manchester: Manchester University Press.

HARRISON, J. (2007): European Broadcasting Law and Policy. Cambridge: Cambridge University Press.

HEROLD, A. (2009): European Film Policies in the Context of EU and International. Groningen: Europa Law Publishing.

HOWLETT, M.; y RAMESH, M. (2003): Studying Public Policy. Policy Cycles and Policy Subsystems. Ontario: Oxford University Press, $2^{\text {nd }}$ edition.

HUMPHREYS, P.: The Principal Axes of the European Union's Audiovisual Policy. En FERNÁNDEZ ALONSO, I.; y MORAGAS, M. (eds.) (2008): Communication and Cultural Policies in Europe. Bellaterra: Universidad Autónoma de Barcelona, pp. 151-184.

- EU Audiovisual Policy, Cultural Diversity and Future of Public Service Broadcasting. En HARRISON, J.; y WESSELS, B. (2008): Mediating Europe. New Media and the European Public Sphere. Londres: Berghahn Books, capítulo 8.

- Regulatory policy and national content. En RABOY, M. (ed.) (2003): L'Avenir de la Réglementation de la Radiodiffusion. Québec: Centre d'études sur les médias - Université Laval, pp. 177-207.

JAKUBOWICZ K.: Media Governance Structures in Europe. En DE BENS, E. (ed.) (2007): Media between Culture and Commerce, Changing Media - Changing Europe Series, volume 4. London: Intellect, pp. 197-223.

KANZLER, M.; y LANGE A. (2008): The place of third country film and audiovisual works in European markets. Strasbourg: European Audiovisual Observatory.

KARIPPINEN, K.: "Media diversity and the politics of criteria. Diversity assessment and technocratisation of European media policy", Nordicom Review, 2006, pp.53-68.

MOSCO, V. (2009): La economía política de la comunicación. Reformulación y renovación. Barcelona: Bosch Comunicación. 
Audiovisual europeo, diversidad cultural y mercado global: Análisis de las actuaciones exteriores de la política audiovisual de la unión europea

MURCIANO, M.: "Los nuevos valores de las políticas de comunicación", Cuadernos de Información, nº18 (2005), pp. 89-98.

MURCIANO, M.; y CRUSAFON, C.: The impact of EU Media Policies abroad: the case of MERCOSUR Audiovisual policy. ECREA (2008): Actas del Congreso Communication Policies and Culture in Europe. Barcelona, 26-28 de noviembre. Barcelona, CD Rom.

PARLAMENTO EUROPEO Y CONSEJO DE LA UNIÓN EUROPEA (2009): DECISION No 1041/2009/EC OF THE EUROPEAN PARLIAMENT AND OF THE COUNCIL of 21 October 2009 establishing an audiovisual cooperation programme with professionals from third countries (MEDIA Mundus), Official Journal of the European Union, L288/10, 4 de noviembre. [Consulta: 30 octubre 2009].

http://eurlex.europa.eu/LexUriServ/LexUriServ.do?uri=OJ:L:2009:288:0010:0017:EN:PDF

- (2008) CARIFORUM - PROTOCOL III: On Cultural Cooperation, Official Journal of the European Union, L 289/II/1938 EN 30.10.2008. [Consulta: 30 octubre 2009].

http://www.sice.org/Trade/CAR_EU_EPA_e/Protocol_III_e.pdf

- (2007): DIRECTIVA 2007/65/CE DEL PARLAMENTO EUROPEO Y DEL CONSEJO de 11 de diciembre de 2007 por la que se modifica la Directiva 89/552/CEE del Consejo sobre la coordinación de determinadas disposiciones legales, reglamentarias y administrativas de los Estados miembros relativas al ejercicio de actividades de radiodifusión televisiva, Diario Oficial de la Unión Europea, L332/27, 18 de diciembre. [Consulta: 1 septiembre 2009].

http://eurlex.europa.eu/LexUriServ/LexUriServ.do?uri=OJ:L:2007:332:0027:0045:ES:PDF)

- (2006): DECISIÓN No 1718/2006/CE DEL PARLAMENTO EUROPEO Y DEL CONSEJO de 15 de noviembre de 2006 relativa a la aplicación de un programa de apoyo al sector audiovisual europeo (MEDIA 2007), Diario Oficial de la Unión Europea, L327/12, 24 de noviembre [Consulta: 1 septiembre 2009].

http://eurlex.europa.eu/LexUriServ/site/es/oj/2006/I 327// 32720061124es00120029.pdf

RECAM (2008): “Cooperación Unión Europea”, Informe de la Secretaría Técnica, Documento interno de la RECAM, junio.

SARIKAKIS, K. (ed.) (2007): Media and Cultural Policy in the European Union. European Studies - An Interdisciplinary Series in European Culture, History and Politics, vol. 24. Amsterdam-New York: Rodopi.

SCHARPF, F.W. (1999): Governing in Europe: Effective or Democratic? Oxford: CUP.

TERZIS, G. (ed.) (2008): European Media governance. The Brussels Dimension. Bristol: Intellect.

WHEELER, M.: "Supranational regulation: television and the European Union", European Journal of Communication, volumen 9, $\mathrm{n}^{\circ} 3$ (2004), pp. 349-369.

Websites de referencia:

Política audiovisual de la Unión Europea:

http://ec.europa.eu/avpolicy/index en.htm

Programa MEDIA INTERNATIONAL:

http://ec.europa.eu/information_society/media/overview/international/index fr.htm

Programa MEDIA MUNDUS:

http://ec.europa.eu/information society/media/overview/media mundus/index fr.htm 
Breve semblanza biográfica de la autora

Carmina Crusafón es Doctora en Ciencias de la Información (UAB, 1999). Profesora Lectora del Departamento de Periodismo y Ciencias de la Comunicación de la Universidad Autónoma de Barcelona. Investigadora especialista en la industria audiovisual y políticas de comunicación, especialmente en la Unión Europea y en América Latina.

(Recibido el 02-02-2010, aceptado el 04-04-2010) 